\title{
A Projection Method for Low Speed Flows
}

\author{
Phillip Colella* and Karen Pao†’ \\ * Department of Mechanical Engineering, University of California, Berkeley, California 94720; and $\dagger$ Scientific \\ Computing Group (CIC-19), Los Alamos National Laboratory, Los Alamos, New Mexico 87545 \\ E-mail: *colella@watt.me.berkeley.edu and †kip@lanl.gov
}

Received November 6, 1997; revised August 4, 1998

\begin{abstract}
We propose a decomposition applicable to low speed, inviscid flows of all Mach numbers less than 1. By using the Hodge decomposition, we may write the velocity field as the sum of a divergence-free vector field and a gradient of a scalar function. Evolution equations for these parts are presented. A numerical procedure based on this decomposition is designed, using projection methods for solving the incompressible variables and a backward-Euler method for solving the potential variables. Numerical experiments are included to illustrate various aspects of our algorithm. (c) 1999 Academic Press
\end{abstract}

Key Words: low speed flows; projection methods; Hodge decomposition.

\section{INTRODUCTION}

We are interested in solving unsteady compressible flow problems where the flow speed is much less than the sound speed and the acoustical waves have large wavelengths. Such problems could be found in a variety of applications such as combustion [1] and flow noise generation [2]. These low speed flows are often difficult to simulate numerically because of the presence of low-amplitude acoustical waves.

One possible way to simulate to low speed flow is to treat such a flow as a fully compressible flow and use an explicit method. These explicit methods have a time step restriction, the CFL condition, which states that for stability, the time step size must be inversely proportional to the maximum of the sum of flow speed and the sound speed. Explicit methods are best suited for problems where the flow speed is on the same order of or larger than the sound speed. In low speed problems, however, the sound speed could be orders of magnitude larger than the flow speed, thus grossly overresolving in time features of the fluid flow.

$\ddagger$ This work was performed under the auspices of the United States Department of Energy by the Los Alamos National Laboratory under Contract W-7405-ENG-36. 
One would then like to somehow separate out the "ill-conditioned" part of the flow and treat it implicitly, while hoping that the rest of the flow may be advanced at an acceptable time step with an economical and accurate method. Such is the approach first taken by Amsden and Harlow [3] and later expanded by many others [4-11]. A common theme in all these methods is splitting: by separating the flow variables into various parts, one may identify the part of the flow that needs implicit treatment and thereby bypass the stringent CFL condition dominated by the sound speed.

In their original paper [3], Amsden and Harlow treated the density variable implicitly; later, it was understood that the pressure variable is the problematic one. Casulli and Greenspan [4] were able to show that only pressure needs to be discretized implicitly, and the resulting difference equations are solved via an iterative procedure. Patnaik et al. [5] extended the work of Casulli and Greenspan to include an "implicitness parameter" to tune the degree of implicitness. Various other formulations have also been proposed in [6-8]. Klein [9] and co-workers [10] have also proposed a numerical method based on asymptotics, which were an extension of the low Mach number asymptotics of Klainermann and Majda [12].

To develop a numerical method useful in the regime of our interest, we shall study a decomposition of the equations of inviscid, compressible flows. These equations will be rewritten in terms of a Hodge decomposition of the velocity field and in terms of auxiliary pressures. With the new equations, we will separate the flow into the divergence-free part, one that varies on a time scale determined by the flow speed, and a part that may contain fast sound waves. The former part may be advanced with time step determined solely by the flow speed. Since the fast sound waves are only present in the latter part, we can advance much of the flow using an explicit method, and apply an implicit method only to the compressible part.

In this paper, we will first present the evolution equations for low speed flows in Section 2. A numerical algorithm for solving these equations will be detailed in Section 3. Results of numerical experiments will be tabulated in Section 4.

\section{THE GOVERNING EQUATIONS}

Consider the inviscid, compressible Euler equations for density $\rho(x, t)$, velocity $u(x, t)$, and pressure $p(x, t)$ in a closed rectangular container solid-wall,

$$
\begin{aligned}
\rho_{t}+\nabla \cdot(\rho \mathbf{u}) & =0, \\
\mathbf{u}_{t}+(\mathbf{u} \cdot \nabla) \mathbf{u}+\frac{1}{\rho} \nabla p & =0, \\
p_{t}+(\mathbf{u} \cdot \nabla) p+\rho c^{2}(\nabla \cdot \mathbf{u}) & =0,
\end{aligned}
$$

with initial values

$$
\begin{aligned}
& \rho(x, 0)=\rho_{0}(x), \\
& \mathbf{u}(x, 0)=\mathbf{u}_{0}(x), \\
& p(x, 0)=p_{0}(x),
\end{aligned}
$$

and the boundary conditions 
Here $c^{2}=\gamma p / \rho$ is the square of sound speed, and $\gamma$ is the ratio of specific heats. We would like to rewrite the Euler equations in terms of a divergence-free velocity field and a "correction." Various asymptotic formulations have been studied by Klainermann and Majda [12] and Kreiss et al. [13]. Instead of an asymptotic expansion, we shall study the exact decomposition of the velocity field.

Our approach is based on the Hodge decomposition (see, for example, [14, Sect. 1.3]). Classically, the Hodge decomposition is a splitting of an arbitrary vector field into two orthogonal components, one divergence-free, and the other the gradient of a scalar field. If $\mathbf{w}=\mathbf{w}(x)$ is a smooth vector field defined on a smooth region $\Omega$, with

$$
\int_{\partial \Omega} \mathbf{w} \cdot \mathbf{n} d S=0,
$$

then $\mathbf{w}$ can be written as

$$
\mathbf{w}=\mathbf{w}_{d}+\frac{1}{\rho} \nabla \phi,
$$

where $\nabla \cdot \mathbf{w}_{d}=0$ and $\mathbf{w}_{d} \cdot \mathbf{n}=0$ on the boundary of $\Omega$, and $\phi$ is the solution to the elliptic equation

$$
\begin{aligned}
L_{\rho} \phi & =\nabla \cdot \mathbf{w} & & \text { in } \Omega, \\
\frac{1}{\rho} \frac{\partial \phi}{\partial n} & =\mathbf{w} \cdot \mathbf{n} & & \text { on } \partial \Omega,
\end{aligned}
$$

with the variable-coefficient second-order elliptic operator

$$
L_{\rho} \phi=\nabla \cdot \frac{1}{\rho} \nabla \phi .
$$

The decomposition is orthogonal with respect to the density-weighted inner product, that is,

$$
\int_{\Omega} \mathbf{w}_{d} \cdot \frac{1}{\rho} \nabla \phi \rho d V=0 .
$$

The Hodge decomposition is unique.

We may define the variable-density projection operators

$$
\begin{aligned}
\mathbf{P}_{\rho} & \equiv I-\mathbf{Q}_{\rho}, \\
\mathbf{Q}_{\rho} & \equiv \frac{1}{\rho} \nabla\left(L_{\rho}^{-1}\right) \nabla \cdot
\end{aligned}
$$

The operator $\mathbf{P}_{\rho}$ is an orthogonal projection operator on $L^{2}$ functions, defined by

$$
\begin{aligned}
& \mathbf{P}_{\rho}=\mathbf{P}_{\rho}^{T}, \\
& \mathbf{P}_{\rho}^{2}=\mathbf{P}_{\rho} .
\end{aligned}
$$

$\mathbf{P}_{\rho}$ takes an $L^{2}$ function to the space of divergence-free functions. 
In terms of the projection operators, the Hodge decomposition is

$$
\mathbf{w}=\mathbf{P}_{\rho} \mathbf{w}+\mathbf{Q}_{\rho} \mathbf{w}
$$

with the divergence-free part

$$
\mathbf{P}_{\rho} \mathbf{w} \equiv \mathbf{w}_{d}
$$

and the gradient

$$
\mathbf{Q}_{\rho} \mathbf{w} \equiv \frac{1}{\rho} \nabla \phi
$$

The constant-density projection operators

$$
\begin{aligned}
\mathbf{P}_{0} & \equiv I-\mathbf{Q}_{0}, \\
\mathbf{Q}_{0} & \equiv \nabla\left(\Delta^{-1}\right)(\nabla \cdot),
\end{aligned}
$$

are defined similarly but with $\rho \equiv 1$. We shall apply these operators to the Euler equations.

Suppose now the flow velocity field $\mathbf{u}$ in the Euler equation is in $L^{2}$; then we may apply Hodge decomposition to $\mathbf{u}$ and split it into a divergence-free part and a curl-free part,

$$
\mathbf{u}=\mathbf{u}_{d}+\mathbf{u}_{p}
$$

where

$$
\begin{aligned}
\mathbf{P}_{0} \mathbf{u} & =\mathbf{u}_{d}, \\
\mathbf{Q}_{0} \mathbf{u} & =\mathbf{u}_{p} .
\end{aligned}
$$

We also define

$$
\mathbf{A}_{d} \mathbf{u} \equiv \mathbf{u} \cdot \nabla \mathbf{u}-\nabla \frac{\left|u_{p}\right|^{2}}{2}
$$

and an "acoustic pressure" as

$$
\delta \equiv p-\pi
$$

where $\pi$ is an auxiliary pressure variable defined by

$$
\frac{1}{\rho} \nabla \pi=-\mathbf{Q}_{\rho} \mathbf{A}_{d} \mathbf{u} .
$$

Finally, we define a potential $\psi$ by

$$
\nabla \psi \equiv-\mathbf{Q}_{0}\left(\frac{1}{\rho} \nabla \delta\right)
$$


We now make the ansatz that, for a closed rectangular container,

$$
\begin{aligned}
\frac{\partial \rho}{\partial t}+\nabla \cdot(\rho \mathbf{u}) & =0 \\
\frac{\partial \mathbf{u}_{d}}{\partial t}+\mathbf{P}_{\rho} \mathbf{A}_{d} \mathbf{u}+\mathbf{P}_{0}\left(\frac{1}{\rho} \nabla \delta\right) & =0 \\
\frac{\partial \mathbf{u}_{p}}{\partial t}+\nabla \frac{\left|u_{p}\right|^{2}}{2}+\mathbf{Q}_{0}\left(\frac{1}{\rho} \nabla \delta\right) & =0 \\
\frac{\partial \delta}{\partial t}+\gamma p \nabla \cdot \mathbf{u}_{p} & =-\left(\mathbf{u} \cdot \nabla p+\frac{\partial \pi}{\partial t}\right),
\end{aligned}
$$

with initial values

$$
\begin{aligned}
\rho(x, 0) & =\rho_{0}(x), \\
\mathbf{u}_{d}(x, 0) & =\mathbf{P}_{0} \mathbf{u}_{0}(x), \\
\mathbf{u}_{p}(x, 0) & =\mathbf{Q}_{0} \mathbf{u}_{0}(x), \\
\delta(x, 0) & =p_{0}(x)+L_{\rho}^{-1}\left(\nabla \cdot\left(\mathbf{u}_{0}(x) \cdot \nabla \mathbf{u}_{0}(x)\right)\right),
\end{aligned}
$$

and the boundary conditions

$$
\begin{aligned}
& \mathbf{u}_{d} \cdot \mathbf{n}=0, \\
& \mathbf{u}_{p} \cdot \mathbf{n}=0 .
\end{aligned}
$$

Note that by summing (12) and (13) we obtain (2), the momentum equation for compressible flow. Also note that (14) is simply the pressure equation (3) rewritten in terms of $\pi$ and $\delta$. The initial conditions and the boundary conditions match those of Euler as well. Since the Hodge decomposition is unique, the $\mathbf{u}_{d}$ and $\mathbf{u}_{p}$ that satisfy Eqs. (11)-(14) must be the unique solution of these equations.

The term $\mathbf{P}_{0}\left(\frac{1}{\rho} \nabla \delta\right)$ represents baroclinic generation of vorticity by the acoustics, that is, a "feedback" mechanism from the acoustics to the fluid flow. In a purely incompressible flow, this feedback is zero. As $M a \rightarrow 0$, Majda and Klainermann [12] have shown that, with initially divergence-free data, $\left|u_{p}\right| /\left|u_{d}\right| \sim O(M a)$, and $|\nabla p| \sim O(M a)$. Terms involving $\mathbf{u}_{p}$ in Eq. (12) become lower order, and $\mathbf{P}_{0}\left(\frac{1}{\rho} \nabla \delta\right) \rightarrow 0$ as well. Thus the incompressible Euler equation (with variable density) is recovered in the zero-Mach number limit, with $\pi$ as the limiting incompressible pressure.

In the zero-Mach number limit, the total pressure may be written as (see [12])

$$
p(x, t)=P_{0}(t)+M a^{2} p_{2}+\cdots
$$

That is, when properly scaled, $\pi \sim O\left(M a^{2}\right)$. In numerical computations one may actually excite the acoustic feedback mechanism by including an $O(M a)$ term in the initial pressure (see [10]). Thus it is imperative that, in a nearly incompressible computation, the evolution equations and the numerical method do not allow such excitations in time.

On the other hand, suppose now that the divergence-free component of the velocity is zero initially, that is, $\mathbf{u}_{d}(x, 0)=0$. Then $\pi=0$ since $\mathbf{A}_{d} \mathbf{u}=0$, and $p \equiv \delta$. For a barotropic fluid, $p=f(\rho)$, and one can show that $\frac{1}{\rho} \nabla \delta$ is a pure gradient, and $\mathbf{P}_{0}\left(\frac{1}{\rho} \nabla \delta\right) \equiv 0$ for all 
times. Thus $\mathbf{u}_{d} \equiv 0$ for all times, and Eq. (13) becomes the evolution equation for $\mathbf{u}$, a purely potential flow. A numerical scheme should preserve this limit as well, by ensuring that the vortical component of the flow (and the associated pressure variables) does not grow appreciably when properly initialized.

\section{THE NUMERICAL METHOD}

The numerical method for solving the initial-value problem (11)-(14) consists of the advection of density and velocity, various discrete projections, and an acoustics solve involving $\mathbf{u}_{p}$ and $\delta$.

We seek the numerical solution of (11)-(14) in a rectangular closed container $[a, b] \times$ $[c, d]$, which is discretized into $M$ intervals in the $x$-direction and $N$ intervals in the $y$ direction, of widths $\Delta x=(b-a) / M$ and $\Delta y=(d-c) / N$, respectively. A time discretization of $\Delta t$ is used. At time $t^{n}=n \Delta t$, we have $\mathbf{u}^{n}=\left(u^{n}, v^{n}\right), \delta^{n}, \rho^{n}, \pi^{n-1 / 2}, \mathbf{u}_{d}^{n}=\left(u_{d}, v_{d}\right)$ at the cell centers $(i, j), i=1 \ldots M, j=1 \ldots N$, and $\mathbf{u}_{p}^{n}=\left(u_{p}^{n}, v_{p}^{n}\right)$ at cell edges $\left(i+\frac{1}{2}, j\right)$ and $\left(i, j+\frac{1}{2}\right)$, respectively.

\subsection{The Discretized Equations}

Equations (11)-(14) are written in the form

$$
V_{t}+B\left(V, V_{x}, V_{y}\right)=F \text {. }
$$

Our approach to solving Eqs. (11)-(14) is to discretize $B$ in space at the appropriate times, and march $V$ in time.

The discretization of $B$ requires the discretizations of the divergence operator $D$ and the gradient operator $G$. Since the values of some of the variables are prescribed at cell centers, while some are prescribed at cell edges, we will need to define $D$ and $G$ that will take the finite differences of $V$ at both cell centers and cell edges and return values at both centers and edges. It turns out that only two divergence and two gradient operators are required. The operator $D^{\text {mac }}$ is the discrete divergence operator applied to edge values, and $D^{0}$ the discrete divergence operator applied to cell-centered values; both operations yield cell-centered scalars. The operator $G^{\mathrm{mac}}$ is the discrete gradient operator, applied to a scalar at the cell centers to yield a vector field at the cell edges, while $G^{0}$ is the discrete gradient operator, applied to a scalar at the cell edges to yield a vector field at the cell centers. These and other spatial operators will be described in detail in Section 3.2.

To advance the numerical solution one time step, we first use a Godunov advection procedure to advance the density $\rho$ to time $t^{n+1}$, and compute the source term $\mathbf{A}_{d}(\mathbf{u})^{n+1 / 2}$ for the projection of the incompressible pressure:

$$
\begin{aligned}
\rho^{n+1} & =\rho^{n}-\Delta t D^{\mathrm{mac}}(\rho \mathbf{u})^{n+1 / 2}, \\
\mathbf{u}_{d}^{*} & =\mathbf{u}_{d}^{n}-\Delta t\left(\mathbf{A}_{d}(\mathbf{u})^{n+1 / 2}+\frac{1}{\rho^{n+1 / 2}} G^{0} \pi^{n-1 / 2}\right) .
\end{aligned}
$$

We then solve a (variable density) Poisson problem for $L_{\rho}$ to obtain the incompressible pressure $\pi^{n+1 / 2}$ :

$$
\pi^{n+1 / 2}=\pi^{n-1 / 2}-L_{\rho^{n+1 / 2}}^{-1} D^{0}\left(\frac{\mathbf{u}_{d}^{*}-\mathbf{u}_{d}^{n}}{\Delta t}\right) .
$$


Next we solve for the acoustic variables $\mathbf{u}_{p}$ and $\delta$ at time $t^{n+1}$ by first discretizing the corresponding differential equations (13) and (14) implicitly:

$$
\begin{aligned}
\mathbf{u}_{p}^{*} & =\mathbf{u}_{p}^{n}-\Delta t\left(G^{\mathrm{mac}}\left(\frac{\left|u_{p}\right|^{2}}{2}\right)^{n}+\frac{1}{\rho^{n}} G^{\mathrm{mac}} \delta^{n+1}\right), \\
\delta^{n+1} & =\delta^{n}-\Delta t\left(\gamma p^{n} D^{\mathrm{mac}} \mathbf{u}_{p}^{*}+(\mathbf{u} \cdot \nabla p)^{n}+\frac{\pi^{n+1 / 2}-\pi^{n-1 / 2}}{\Delta t}\right) .
\end{aligned}
$$

Solving these equations algebraically for $\delta^{n+1}$, we obtain a Helmholtz equation for $\delta^{n+1}$. Once we solve the Helmholtz equation for $\delta^{n+1}$, we perform another projection to obtain $\mathbf{u}_{p}^{n+1}$ :

$$
\mathbf{u}_{p}^{n+1}=\mathbf{u}_{p}^{n}-\Delta t\left(G^{\mathrm{mac}}\left(\frac{\left|u_{p}\right|^{2}}{2}\right)^{n}+\mathbf{Q}_{0}^{\mathrm{mac}}\left(\frac{1}{\rho^{n+1}} G^{\mathrm{mac}} \delta^{n+1}\right)\right) .
$$

Simultaneously to the above steps, we collect the terms needed to advance the incompressible velocity $\mathbf{u}_{d}$ to time $t^{n+1}$ :

$$
\mathbf{u}_{d}^{n+1}=\mathbf{u}_{d}^{*}-\Delta t\left(\frac{1}{\rho^{n+1}} G^{0}\left(\pi^{n+1 / 2}-\pi^{n-1 / 2}\right)+\mathbf{P}_{0}^{0}\left(\frac{1}{\rho^{n+1}} G^{0} \delta^{n+1}\right)\right) .
$$

In the next few sections, we shall describe the implementations of the above scheme in greater detail. First, in Section 3.2, we describe the spatial discretizations of the discrete divergence $(D)$ and gradient $(G)$ operators $D^{\text {mac }}, D^{0}, G^{\text {mac }}$, and $G^{0}$, as well as the discretizations of the projection operators $\mathbf{P}$ and $\mathbf{Q}$ and the operator $L_{\rho}$. Then, in Section 3.3, we describe the time stepping of the above scheme in more depth. Inflow and outflow boundary conditions used in the numerical experiments are explained in Section 3.4.

\subsection{Spatial Discretizations}

We shall need to determine various divergence and gradient operators, as well as the appropriate discretization of the projection operators $\mathbf{P}$ and $\mathbf{Q}$, and the discretization of the operator $L_{\rho}$.

Suppose $D$ and $G$ are the discrete approximations to the divergence and gradient operators; then we would like, for an arbitrary vector field $\mathbf{w}=\left(w^{1}, w^{2}\right)$ on a region $\Omega$, to have

$$
\mathbf{w}=\mathbf{w}_{d}+\frac{1}{\rho} G \phi
$$

where

$$
D \mathbf{w}_{d}=0,
$$

and $\phi$ satisfies

$$
\begin{aligned}
L_{\rho} \phi & =D \mathbf{w} & & \text { in } \Omega, \\
\mathbf{n} \cdot G \phi & =0 & & \text { on } \partial \Omega .
\end{aligned}
$$


Ideally, we would like to have $D=\left(-\frac{1}{\rho} G\right)^{T}$; that is, the divergence and gradient are adjoint of each other with respect to the density-weighted inner product. The discretization of $L_{\rho}$ is given by $L_{\rho}^{h}=D \frac{1}{\rho} G$. Note that because of the adjoint property, $\phi$ as defined above can always be found (up to a constant).

Recall that the continuous projection operator $\mathbf{P}_{\rho}$ is self-adjoint $\left(\mathbf{P}_{\rho}=\mathbf{P}_{\rho}^{T}\right)$ and idempotent $\left(\mathbf{P}_{\rho}^{2}=\mathbf{P}_{\rho}\right)$. We would like our discretization of the projection operator $\mathbf{P}^{h}$ to have these properties. If

$$
\mathbf{P}^{h}=I-\frac{1}{\rho} G\left(D \frac{1}{\rho} G\right)^{-1} D
$$

with $D$ and $G$ defined as above, then these properties of the projection operators are satisfied. This projection operator, however, is not suitable for computation. The biggest disadvantage is that the discrete operator $D \frac{1}{\rho} G$ would have a null space of four dimensions (see [15]), and the solution decouples into four independent components. This is the so-called "checkerboard effect," or alternating modes.

Instead, we use the projection operator

$$
\mathbf{P}^{h}=I-\frac{1}{\rho} G\left(L_{\rho}^{h}\right)^{-1} D .
$$

There is no decoupling of the solution. However,

$$
L_{\rho}^{h} \neq D \frac{1}{\rho} G .
$$

This causes the discrete projection operator $\mathbf{P}^{h}$ to be non-idempotent. We call this an approximate projection.

In the algorithm specified above, we use two different discretizations of the gradient operator. One discretization of $G$, denoted by $G^{0}$, is based on centered differencing. The other, $G^{\text {mac }}$, uses the staggered marker-and-cell (MAC) mesh of Harlow and Welch [16]. On a rectangular grid, the MAC gradient operator is defined by the differences of cell-centered values:

$$
\begin{aligned}
\left(G^{\mathrm{mac}} \phi\right)_{i+1 / 2, j}^{x} & =\frac{\phi_{i+1, j}-\phi_{i, j}}{\Delta x}, \\
\left(G^{\mathrm{mac}} \phi\right)_{i+1 / 2, j}^{y} & =\frac{\phi_{i+1, j+1}-\phi_{i+1, j-1}+\phi_{i, j+1}-\phi_{i, j-1}}{4 \Delta y}, \\
\left(G^{\mathrm{mac}} \phi\right)_{i, j+1 / 2}^{x} & =\frac{\phi_{i+1, j+1}-\phi_{i-1, j+1}+\phi_{i+1, j}-\phi_{i-1, j}}{4 \Delta x}, \\
\left(G^{\mathrm{mac}} \phi\right)_{i, j+1 / 2}^{y} & =\frac{\phi_{i, j+1}-\phi_{i, j}}{\Delta y} .
\end{aligned}
$$

The cell-centered gradient operator may be defined in terms of the MAC gradient operator:

$$
\begin{aligned}
\left(G^{0} \phi\right)_{i, j}^{x} & =\frac{1}{2}\left(\left(G^{\mathrm{mac}} \phi\right)_{i+1 / 2, j}^{x}+\left(G^{\mathrm{mac}} \phi\right)_{i-1 / 2, j}^{x}\right) \\
& =\frac{1}{2} \frac{\left(\phi_{i+1, j}-\phi_{i-1, j}\right)}{\Delta x} .
\end{aligned}
$$




$$
\begin{aligned}
\left(G^{0} \phi\right)_{i, j}^{y} & =\frac{1}{2}\left(\left(G^{\mathrm{mac}} \phi\right)_{i, j+1 / 2}^{y}+\left(G^{\mathrm{mac}} \phi\right)_{i, j-1 / 2}^{y}\right) \\
& =\frac{1}{2} \frac{\left(\phi_{i, j+1}-\phi_{i, j-1}\right)}{\Delta y} .
\end{aligned}
$$

The divergence of a vector field shall always he defined at the cell centers. Since we could take the divergence of either a cell-centered vector field or an edge-centered vector field, we shall need two kinds of divergence operators as well: The edge-centered divergence is

$$
\left(D^{\mathrm{mac}} \mathbf{w}\right)_{i, j}=\frac{w_{i+1 / 2, j}^{1}-w_{i-1 / 2, j}^{1}}{\Delta x}+\frac{w_{i, j+1 / 2}^{2}-w_{i, j-1 / 2}^{2}}{\Delta y},
$$

and the cell-centered divergence is

$$
\left(D^{0} \mathbf{w}\right)_{i, j}=\frac{1}{2}\left(\frac{w_{i+1, j}^{1}-w_{i-1, j}^{1}}{\Delta x}+\frac{w_{i, j+1}^{2}-w_{i, j-1}^{2}}{\Delta y}\right) .
$$

Note that the cell-centered divergence can also be viewed as an average of the edge-centered divergence, if we let

$$
\begin{aligned}
& w_{i+1 / 2, j}^{1}=\frac{1}{2}\left(w_{i+1, j}^{1}+w_{i, j}^{1}\right), \\
& w_{i, j+1 / 2}^{2}=\frac{1}{2}\left(w_{i, j+1}^{2}+w_{i, j}^{2}\right),
\end{aligned}
$$

that is, interpolate the edge-centered values by averaging the cell-centered values.

Boundary conditions are applied to the gradients by using values in the "ghost cells," cells outside the computational domain. For example, at the left edge, we would have

$$
\left(G^{0} \phi\right)_{1, j}^{x}=\frac{1}{2} \frac{\left(\phi_{2, j}-\phi_{0, j}\right)}{\Delta x}
$$

where $\phi_{0, j}$ is in the first row of ghost cells, its value determined by a third-order extrapolation:

$$
\phi_{0, j}=3 \phi_{1, j}-3 \phi_{2, j}+\phi_{3, j}
$$

Boundary conditions are applied to the divergences by considering the fluxes at the boundary. We can write $D^{0} \mathbf{w}$ in conservation form,

$$
\left(D^{0} \mathbf{w}\right)_{i, j}=\frac{F_{i+1 / 2, j}-F_{i-1 / 2, j}}{\Delta x}+\frac{F_{i, j+1 / 2}-F_{i, j-1 / 2}}{\Delta y},
$$

with

$$
\begin{aligned}
& F_{i+1 / 2, j}=\frac{1}{2}\left(w_{i+1, j}^{1}+w_{i, j}^{1}\right) \\
& F_{i, j+1 / 2}=\frac{1}{2}\left(w_{i, j+1}^{2}+w_{i, j}^{2}\right)
\end{aligned}
$$

for $i=1, \ldots, M, j=1, \ldots, N$. Then the boundary condition is applied to $F$ on the boundary. For example, on the left boundary, the condition $\mathbf{w} \cdot \mathbf{n}=h$ is enforced by setting $F_{1 / 2, j}=h_{1 / 2, j}$. 
The discrete operator $L_{\rho}^{h}$ is given by

$$
\begin{aligned}
\left(L_{\rho}^{h} \phi\right)_{i, j} \equiv & D^{\mathrm{mac}} \frac{1}{\rho} G^{\mathrm{mac}} \phi_{i, j} \\
= & \frac{1}{\Delta x}\left\{\frac{1}{\rho_{i+1 / 2, j}} \frac{\phi_{i+1, j}-\phi_{i, j}}{\Delta x}-\frac{1}{\rho_{i-1 / 2, j}} \frac{\phi_{i, j}-\phi_{i-1, j}}{\Delta x}\right\} \\
& +\frac{1}{\Delta y}\left\{\frac{1}{\rho_{i, j+1 / 2}} \frac{\phi_{i, j+1}-\phi_{i, j}}{\Delta y}-\frac{1}{\rho_{i, j-1 / 2}} \frac{\phi_{i, j}-\phi_{i, j-1}}{\Delta y}\right\} .
\end{aligned}
$$

The inverse density, evaluated at the cell edges, is obtained by the average of the inverse densities:

$$
\begin{aligned}
& \frac{1}{\rho_{i+1 / 2, j}}=\frac{1}{2}\left(\frac{1}{\rho_{i+1, j}}+\frac{1}{\rho_{i, j}}\right) ; \\
& \frac{1}{\rho_{i, j+1 / 2}}=\frac{1}{2}\left(\frac{1}{\rho_{i, j+1}}+\frac{1}{\rho_{i, j}}\right) .
\end{aligned}
$$

In the rectangular closed container, we have the Neumann boundary condition

$$
\frac{\partial \phi}{\partial n}=g \quad \text { on } \partial \Omega
$$

On the left boundary, for example, we have

$$
\frac{\left(\phi_{1, j}-\phi_{0, j}\right)}{\Delta x}=g_{1 / 2, j},
$$

or

$$
\phi_{0, j}=\phi_{1, j}-\Delta x g_{1 / 2, j}
$$

And thus $L_{\rho} \phi$ can be computed for all cells in the computational domain.

The projection operators can now be defined:

$$
\begin{aligned}
\mathbf{Q}_{\rho}^{\mathrm{mac}} & =\frac{1}{\rho} G^{\mathrm{mac}}\left(L_{\rho}^{h}\right)^{-1} D^{\mathrm{mac}} ; \\
\mathbf{P}_{\rho}^{\mathrm{mac}} & =I-\mathbf{Q}_{\rho}^{\mathrm{mac}} ; \\
\mathbf{Q}_{\rho}^{0} & =\frac{1}{\rho} G^{0}\left(L_{\rho}^{h}\right)^{-1} D^{0} ; \\
\mathbf{P}_{\rho}^{0} & =I-\mathbf{Q}_{\rho}^{0} .
\end{aligned}
$$

There are two kinds of projection operators, a cell-centered projection and a MAC projection. The difference is in the divergence and gradient operators used. In applying the MAC projection $\mathbf{P}_{\rho}^{\mathrm{mac}}$ to a vector field $\mathbf{w}$, we solve the Poisson problem

$$
L_{\rho}^{h} \phi=D^{\mathrm{mac}} \mathbf{w}
$$


with the appropriate boundary conditions. Then

$$
\mathbf{Q}_{\rho}^{\mathrm{mac}} \mathbf{w}=\frac{1}{\rho} G^{\mathrm{mac}} \phi
$$

On the other hand, in a cell-centered projection $\mathbf{P}_{\rho}^{0} \mathbf{w}$, one would solve the Poisson problem

$$
L_{\rho} \phi=D^{0} \mathbf{w}
$$

with the appropriate boundary conditions. Then

$$
\mathbf{Q}_{\rho}^{0} \mathbf{w}=\frac{1}{\rho} G^{0} \phi
$$

The Laplacian operators in the two projections are the same, but the arguments of the divergence operators in the right hand side of the Poisson problems reside at different places on the grid: in the regular projection, $\mathbf{w}$ is at the cell centers; in the MAC projection, $\mathbf{w}$ is staggered. In both projections, the potential $\phi$ will be cell-centered, but the final projected vector fields will again reside at different places on the grid.

\subsection{Details of the Time Stepping}

As mentioned above, to advance the solution one time step, we compute (15)-(21) roughly in that order. Here we shall present the details of time advancement.

First we need to compute $\mathbf{A}_{d} \mathbf{u}$ at the cell centers from the equation for $\mathbf{u}_{d}$ (16), while advancing $\rho$ conservatively to the time $t^{n+1}$ using the density equation (11). This may be accomplished by a Godunov-type procedure. In our Godunov procedure, we first extrapolate the values at cell centers to the edges and at half time, using Taylor series. For example, on the vertical edge $\left(i+\frac{1}{2}, j\right)$, we may extrapolate from the cell on the left:

$$
\mathbf{u}_{d i+1 / 2, j}^{n+1 / 2, L} \approx \mathbf{u}_{d i, j}^{n}+\frac{\Delta x}{2} \frac{\partial \mathbf{u}_{d}^{n}}{\partial x}+\frac{\Delta t}{2} \frac{\partial \mathbf{u}_{d}^{n}}{\partial t},
$$

with the partial derivatives evaluated at cell $(i, j)$, or we may extrapolate from the cell on the right,

$$
\mathbf{u}_{d i+1 / 2, j}^{n+1 / 2, R} \approx \mathbf{u}_{d i+1, j}^{n}-\frac{\Delta x}{2} \frac{\partial \mathbf{u}_{d}^{n}}{\partial x}+\frac{\Delta t}{2} \frac{\partial \mathbf{u}_{d}^{n}}{\partial t},
$$

with the partial derivatives evaluated at cell $(i+1, j)$. In both expressions we then replace the time derivative by employing the equation

$$
\frac{\partial \mathbf{u}_{d}}{\partial t}=-\left((\mathbf{u} \cdot \nabla) \mathbf{u}_{d}+\left(\mathbf{u}_{d} \cdot \nabla\right) \mathbf{u}_{p}\right)-\frac{1}{\rho} \nabla \delta-\nabla \psi .
$$

This is simply Eq. (16) but with the term

$$
\mathbf{Q}_{\rho}\left(\mathbf{A}_{d} \mathbf{u}\right)
$$

omitted. 
Thus, at the edge $\left(i+\frac{1}{2}, j\right)$, for the first component of velocity,

$$
\begin{aligned}
u_{d}^{n+1 / 2, L}= & u_{d i, j}^{n}+\frac{\Delta x}{2} \frac{\partial u_{d}}{\partial x}-\frac{\Delta t}{2}\left(u_{i, j} \frac{\partial u_{d}}{\partial x}+v_{i, j} \frac{\partial u_{d}}{\partial y}+u_{i, j} \frac{\partial u_{p}}{\partial x}+v_{i, j} \frac{\partial u_{p}}{\partial y}\right) \\
& -\frac{\Delta t}{2}\left(\frac{1}{\rho} \nabla \delta-\nabla \psi\right) .
\end{aligned}
$$

The spatial derivatives of the velocity $\mathbf{u}_{d}$ in the normal direction is approximated by the limited difference, using the van Leer limiter [18]: let

$$
\begin{aligned}
\Delta^{x} u^{C} & =\frac{1}{2}\left(u_{d i+1, j}-u_{d i-1, j}\right), \\
\Delta^{x} u^{L} & =2\left(u_{d i, j}-u_{d i-1, j}\right), \\
\Delta^{x} u^{R} & =2\left(u_{d i+1, j}-u_{d i, j}\right) .
\end{aligned}
$$

Then

$$
\begin{aligned}
\Delta u_{d i, j} & =\min \left(\left|\Delta^{x} u^{C}\right|,\left|\Delta^{x} u^{L}\right|,\left|\Delta^{x} u^{R}\right|\right) \operatorname{sign}\left(\Delta^{x} u^{C}\right), & & \text { if } \Delta^{x} u^{L} \Delta^{x} u^{R}>0, \\
& =0 & & \text { otherwise. }
\end{aligned}
$$

The derivatives in the transverse direction are upwinded:

$$
\begin{aligned}
\frac{\partial u_{d}}{\partial y} & =\left(u_{d i, j}-u_{d i, j-1}\right) / \Delta y, \quad v_{i, j} \geq 0, \\
& =\left(u_{d i, j+1}-u_{d i, j}\right) / \Delta y, \quad v_{i, j}<0 .
\end{aligned}
$$

The derivative of $u_{p}$ in cell $(i, j)$ in the $x$-direction can be computed easily, since $u_{p}$ are defined on the edges $\left(i+\frac{1}{2}, j\right)$ :

$$
\frac{\partial u_{p}}{\partial x}=\left(u_{p i+1 / 2, j}-u_{p i-1 / 2, j}\right) / \Delta x
$$

and the derivative of $u_{p}$ in cell $(i, j)$ in the $y$-direction is averaged:

$$
\frac{\partial u_{p}}{\partial y}=\frac{1}{4}\left(u_{p i+1 / 2, j+1}-u_{p i+1 / 2, j-1}+u_{p i-1 / 2, j+1}-u_{p i-1 / 2, j-1}\right) / \Delta y .
$$

The derivatives of $\delta$ and $\psi$ are approximated by centered differences.

Once $\mathbf{u}_{d i+1 / 2, j}^{n+1 / 2, L}$ and $\mathbf{u}_{d i+1 / 2, j}^{n+1 / 2, R}$ have been constructed, the ambiguity at the edge $\left(i+\frac{1}{2}, j\right)$ is resolved by choosing the upwind value based on the total velocity normal to the edge $\left(i+\frac{1}{2}, j\right)$,

$$
\begin{aligned}
U_{d i+1 / 2, j}^{n+1 / 2} & =U_{d i+1 / 2, j}^{n+1 / 2, L} & & \text { if } u_{i, j}^{n}+u_{i+1, j}^{n}>0 ; \\
& =U_{d i+1 / 2, j}^{n+1 / 2, R} & & \text { if } u_{i, j}^{n}+u_{i+1, j}^{n}<0 ; \\
& =\frac{1}{2}\left(U_{d i+1 / 2, j}^{n+1 / 2, R}+U_{d i+1 / 2, j}^{n+1 / 2, L}\right) & & \text { if } u_{i, j}^{n}+u_{i+1, j}^{n}=0
\end{aligned}
$$

for $U=u, v$. A similar procedure is applied to find $\mathbf{u}_{d i, j+1 / 2}^{n+1 / 2}$. 
Once the edge velocities at half time are obtained, we correct for the pressure gradient term by applying a MAC projection [21] to the edge velocity values: recall that in the advection step above, we have omitted the term that corresponds to

$$
\frac{\Delta t}{2 \rho} \nabla \pi
$$

The effect of this omission is that the current value of $\mathbf{u}_{d}$ on the edges, denoted here by $\mathbf{u}_{d}^{\text {predicted }}$, is not divergence free. If we write

$$
\mathbf{u}_{d}^{\text {predicted }}=\mathbf{u}_{d}+\nabla \varphi
$$

where $\mathbf{u}_{d}$ is the divergence-free edge value, then a correction for $\mathbf{u}_{d}^{\text {predicted }}$ may be found by solving a Poisson equation for $\varphi$. The Poisson operator in the equation above may be discretized by the same five-point operator $L$, but the source term is formed by taking the MAC divergence of $\mathbf{u}_{d}^{\text {predicted }}$.

Once we solve for $\varphi$, we can correct the predicted values of $\mathbf{u}_{d}$ by taking the discrete gradient of $\varphi$ :

$$
\mathbf{u}_{d}^{n+1 / 2}=\mathbf{u}_{i+1 / 2, j}^{n+1 / 2, \text { predicted }}-G^{\mathrm{mac}} \varphi .
$$

With the corrected $\mathbf{u}_{d}$ on the edges, $\left(\mathbf{A}_{d} \mathbf{u}\right)_{i, j}^{n+1 / 2}$ may now be approximated by finite differences.

We also construct $\rho$ using the Godunov procedure outlined above, at the edges at the half time. To determine the unique edge value at each edge, the density is advected passively by the normal velocity:

$$
\begin{aligned}
\rho_{i+1 / 2, j}^{n+1 / 2} & =\rho_{i+1 / 2, j}^{n+1 / 2, L} & & \text { if } u_{i, j}^{n}+u_{i+1, j}^{n}>0 ; \\
& =\rho_{i+1 / 2, j}^{n+1 / 2, R} & & \text { if } u_{i, j}^{n}+u_{i+1, j}^{n}<0 ; \\
& =\frac{1}{2}\left(\rho_{i+1 / 2, j}^{n+1 / 2, R}+\rho_{i+1 / 2, j}^{n+1 / 2, L}\right) & & \text { if } u_{i, j}^{n}+u_{i+1, j}^{n}=0 .
\end{aligned}
$$

Likewise, $\rho_{i, j+1 / 2}^{n+1 / 2}$ is passively advected by $v^{n}$. The density $\rho$ can now be advanced to the time level $n+1$ conservatively:

$$
\begin{aligned}
\rho^{n+1}= & \rho^{n}-\frac{\Delta t}{\Delta x}\left(\rho_{i+1 / 2, j}^{n+1 / 2} u_{i+1 / 2, j}^{n+1 / 2}-\rho_{i-1 / 2, j}^{n+1 / 2} u_{i-1 / 2, j}^{n+1 / 2}\right) \\
& -\frac{\Delta t}{\Delta y}\left(\rho_{i, j+1 / 2}^{n+1 / 2} v_{i, j+1 / 2}^{n+1 / 2}-\rho_{i, j-1 / 2}^{n+1 / 2} v_{i, j-1 / 2}^{n+1 / 2}\right) .
\end{aligned}
$$

The density $\rho^{n+1 / 2}$, needed in the projection in (17), is a by-product of the Godunov procedure, and already exists on the cell edges. The cell-centered quantity $\mathbf{u}_{d}^{*}$ may also be formed using (16), and $\mathbf{u}_{d}^{n+1}$ may be updated.

According to (17), we obtain $\pi^{n+1 / 2}$ by solving the Poisson problem

$$
L_{\rho^{n+1 / 2}} \delta \pi^{n+1 / 2}=-D^{0}\left(\mathbf{A}_{d} \mathbf{u}^{n+1 / 2}+\frac{1}{\rho^{n+1 / 2}} G^{0} \pi^{n-1 / 2}\right),
$$


with the boundary conditions

$$
\frac{\partial}{\partial n} \delta \pi^{n+1 / 2}=0
$$

at solid wall boundaries. Then

$$
\pi^{n+1 / 2}=\pi^{n-1 / 2}+\delta \pi^{n+1 / 2} .
$$

Once we obtain $\pi^{n+1 / 2}$, the second term on the right hand side of (21) may be computed and $\mathbf{u}_{d}^{n+1}$ may again be updated.

Up to this point, the all-speed algorithm reduces to the conventional projection method (see [19], [20], or [21], for example) for incompressible flows, if incompressible initial data is provided.

To compute the effects of compressibility, we first need to estimate the acoustic source term in (14). We approximate the time derivative of $\pi$ at time level $n$ by

$$
\frac{\partial}{\partial t} \pi^{n} \approx \frac{\pi^{n+1 / 2}-\pi^{n-1 / 2}}{\Delta t} .
$$

We may approximate $(\mathbf{u} \cdot \nabla p)^{n}$ by taking the centered differences of $p^{n}$ in the $x$ - and the $y$-directions. Thus the forcing term is

$$
f_{i, j}^{n}=-\frac{\pi^{n+1 / 2}-\pi^{n-1 / 2}}{\Delta t}-\left(u_{i, j}^{n} \frac{p_{i+1, j}^{n}-p_{i-1, j}^{n}}{2 \Delta x}+v_{i, j}^{n} \frac{p_{i, j+1}^{n}-p_{i, j-1}^{n}}{2 \Delta y}\right) .
$$

Now (18) and (19) may be solved. These two equations are an implicit discretization of (13) and (14). The term $\mathbf{Q}_{0}\left(\frac{1}{\rho} \nabla \delta\right)$ in (13) is "predicted" by $\frac{1}{\rho} \nabla \delta$ in the discrete equation (18), and will be corrected for when we solve the discrete equation (20). Equations (18) and (19) are solved by first solving for $\delta^{n+1}$ algebraically. This gives a Helmholtz equation for $\delta^{n+1}$,

$$
\left(I-\Delta t^{2} \gamma p^{n} L_{\rho^{n}}^{h}\right) \delta^{n+1}=\delta^{n}+\Delta t f_{i, j}^{n}-\Delta t \gamma p^{n}\left(D^{\mathrm{mac}} \mathbf{u}_{p}^{n}-\Delta t L_{0}\left(\frac{\left|u_{p}^{n}\right|^{2}}{2}\right)^{n}\right),
$$

with the boundary conditions

$$
\frac{\partial \delta}{\partial n}=0
$$

This linear system is always diagonally dominant and nonsingular, since $\rho c^{2}=\gamma p>0$. The solution $\delta^{n+1}$ can always be found.

We now correct $\mathbf{u}_{p}^{*}$ by projecting out the part of $\frac{1}{\rho} \nabla \delta^{n+1}$ that is not curl-free. This is accomplished by first solving

$$
\nabla \psi^{n+1}=\mathbf{Q}_{0}^{\operatorname{mac}}\left(\frac{1}{\rho^{n+1}} G^{\mathrm{mac}} \delta^{n+1}\right)
$$

with Neumann boundary condition on all physical boundaries

$$
{\frac{\partial \psi^{n+1}}{\partial n}}^{n}=-\frac{1}{\rho^{n+1}} \frac{\partial \delta}{\partial n}^{n+1}
$$


and updating $\mathbf{u}_{p}$ using (20):

$$
\mathbf{u}_{p}^{n+1}=\mathbf{u}_{p}^{n}-\Delta t\left(G^{\mathrm{mac}}\left(\frac{\left|u_{p}^{n}\right|^{2}}{2}\right)-G^{\mathrm{mac}} \psi^{n+1}\right) .
$$

Now we may compute the last term on the right hand side of (21) by averaging $G^{\mathrm{mac}} \psi^{n+1}$ to the cell centers to form $\mathbf{Q}_{0}^{0}\left(\frac{1}{\rho} G^{0} \delta\right)$. This completes the update of $\mathbf{u}_{d}^{n+1}$.

Since equations involving the sound speed are solved implicitly (in the Helmholtz solve), we no longer need to take sound speed into account in determining a time step that will render the scheme stable. Thus we may choose the time step $\Delta t$ by considering $\mathbf{u}$ only:

$$
\Delta t<C_{c f l} \frac{\min (\Delta x, \Delta y)}{\max _{x}\left(\left|u_{0}(x)\right|,\left|v_{0}(x)\right|\right)} .
$$

The same $\Delta t$ may be used throughout the entire course of the computation; with some modification to the computation of the acoustic source term, one may also use variable time-stepping. A safety factor, $C_{c f l}<1$, is also used.

\subsection{Inflow-Outflow Boundary Conditions}

In choosing appropriate boundary conditions for problems involving inflow and outflow, we are guided by the definition of the Hodge decomposition, namely that the vector field may be decomposed into orthogonal parts, one divergence-free, and one potential, and the divergence-free part is parallel to the boundary near the boundary.

At a solid wall or an inflow boundary, one requires

$$
\mathbf{u} \cdot \mathbf{n}=g
$$

for the inviscid Euler equations. We shall require

$$
\begin{aligned}
& \mathbf{u}_{d} \cdot \mathbf{n}=0 \\
& \mathbf{u}_{p} \cdot \mathbf{n}=g .
\end{aligned}
$$

We shall only consider outflow boundaries where the flow is uniform in the $x$-direction. Thus

$$
v=v_{p}=v_{d} \equiv 0
$$

The value of the potential velocity $u_{p}$ at the outflow can be deduced from the gradient of $\psi$, that is,

$$
u_{p}=\left(G^{\mathrm{mac}}\right)^{x} \psi
$$

at the outflow. The value of $\psi$ in the first ghost cell is needed. For example, if the outflow boundary is on the right, then

$$
\psi_{M+1, j}=3 \psi_{M, j}-3 \psi_{M-1, j}+\psi_{M-2, j}
$$


The vortical component of the velocity, $u_{d}$, must be divergence-free at the boundary. A Neumann boundary condition at $u_{d}$,

$$
u_{d M+1, j}=u_{d M, j},
$$

will be sufficient. Alternatively, a second-order extrapolation

$$
u_{d M+1, j}=2 u_{d M, j}-u_{d M-1, j}
$$

will also yield divergence-free $u_{d}$ at the outflow boundary.

For the projection

$$
\mathbf{P}_{\rho}\left(-\mathbf{u} \cdot \nabla \mathbf{u}+\nabla \frac{\left|u_{p}\right|^{2}}{2}\right)
$$

we may write

$$
-\mathbf{u} \cdot \nabla \mathbf{u}+\nabla \frac{\left|u_{p}\right|^{2}}{2}=\mathbf{P}_{\rho}\left(-\mathbf{u} \cdot \nabla \mathbf{u}+\nabla \frac{\left|u_{p}\right|^{2}}{2}\right)+\frac{1}{\rho} \nabla \pi .
$$

The orthogonality condition gives

$$
\mathbf{n} \cdot \mathbf{P}_{\rho}\left(-\mathbf{u} \cdot \nabla \mathbf{u}+\nabla \frac{\left|u_{p}\right|^{2}}{2}\right)=0
$$

or

$$
\mathbf{n} \cdot\left(-\mathbf{u} \cdot \nabla \mathbf{u}+\nabla \frac{\left|u_{p}\right|^{2}}{2}\right)=\frac{1}{\rho} \frac{\partial \pi}{\partial n}
$$

Note that if one only considers the orthogonality condition, then

$$
\pi=0
$$

is also a valid boundary condition. In our numerical examples, we use (31) for inflow and solid wall boundaries, while (32) is used at the outflow boundary.

Assuming that the inflow quantity $g$ is constant in time, we may determine the boundary condition for the projection

$$
\mathbf{Q}_{0}\left(\frac{1}{\rho} \nabla \delta\right)
$$

which appears in the evolution equation for $\mathbf{u}_{p},(13)$. Let

$$
\mathbf{Q}_{0} \frac{1}{\rho} \nabla \delta \equiv-\nabla \psi
$$

To ascertain

$$
\frac{\partial}{\partial t} \mathbf{u}_{p} \cdot \mathbf{n}=0
$$


we must have

$$
\begin{aligned}
\frac{\partial \psi}{\partial n} & =\frac{\partial}{\partial n}\left(\frac{\left|u_{p}\right|^{2}}{2}\right) \\
& =-\frac{1}{\rho} \frac{\partial \delta}{\partial n} .
\end{aligned}
$$

Thus the boundary conditions for the pressure variable $\delta$ at inflow and walls will be

$$
\frac{\partial \delta}{\partial n}=-\rho \frac{\partial}{\partial n}\left(\frac{\left|u_{p}\right|^{2}}{2}\right) .
$$

At outflow boundary, we shall specify the value of $\delta$, which is equal to the total pressure at outflow. For the $\mathbf{Q}_{0}$ computation,

$$
\frac{\partial \psi}{\partial n}=-\frac{1}{\rho} \frac{\partial \delta}{\partial n}
$$

shall provide a Neumann boundary condition for all boundaries; alternatively,

$$
\frac{\partial \psi}{\partial n}=\frac{\partial}{\partial n}\left(\frac{\left|u_{p}\right|^{2}}{2}\right)
$$

will ensure $\mathbf{u}_{p} \cdot \mathbf{n}=g$ at walls and inflow and may be used at those boundaries.

\section{NUMERICAL EXPERIMENTS}

First we shall present some convergence results. Our first problem is a convergence test in the limit $M a \rightarrow 0$.

The initial flow is a "vortex-in-a-box," a swirly flow with

$$
\begin{aligned}
& u(x, y, 0)=2 \sin ^{2}(\pi x) \sin (\pi y) \cos (\pi y), \\
& v(x, y, 0)=-2 \sin (\pi x) \cos (\pi x) \sin ^{2}(\pi y) ; \\
& p(x, y, 0)=P_{0}, \quad P_{0}=1,10,100,1000 ; \\
& \rho(x, y, 0)=1-\frac{1}{2} \tanh \left(y-\frac{1}{2}\right)
\end{aligned}
$$

on the square $0 \leq x \leq 1,0 \leq y \leq 1$, with the no-flow boundary condition

$$
\mathbf{u} \cdot \mathbf{n}=0
$$

at the walls. We discretize the computational region by

$$
\Delta x=\Delta y=1 / 32,1 / 64,1 / 128,1 / 256
$$

For convergence test purposes, set $\Delta t=0.5 \Delta x$, and advance the solution up to $t=0.125$. Table I shows the range of Mach numbers for which we have done convergence studies. Here the Mach number is determined by taking the maximum of the ratio of the magnitude of the total velocity to the sound speed at $t=0.125$. 


\section{TABLE I}

Mach Numbers $M a$ as a Function of Initial Pressure $\boldsymbol{P}_{0}$

\begin{tabular}{rc}
\hline$P_{0}$ & Approx. Ma \\
\hline 1 & 0.88 \\
10 & 0.28 \\
100 & 0.089 \\
1000 & 0.027 \\
\hline
\end{tabular}

We perform a convergence study. The pointwise error $e^{h}$ is obtained by taking the absolute value of the difference of the computed solution with spacing $h$ and that with spacing $h / 2$. We expect that

$$
e^{h}=C_{p} h^{p}+O\left(h^{p+1}\right)
$$

where $p$ is the order of accuracy, or the rate of convergence. Thus $p$ may be computed from the ratio of $e^{h}$ and $e^{2 h}$ :

$$
p=\log _{2} \frac{e^{2 h}}{e^{h}} .
$$

Tables II-VI show the errors $e^{h}$ and the convergence rates for various discretizations for the density $\rho$, incompressible velocity $u_{d}$, total velocity $u$, incompressible pressure $\pi$, and acoustic pressure $\delta$. For $P=1000$, the convergence rates for the "incompressible" variables $\left(u_{d}, \pi\right)$ are basically second-order. This is actually quite remarkable, considering that our scheme is a mixture of first- and second-order approximations. An examination of the convergence rates for $u, \rho$, and $\delta$ reveals the effect of the backward Euler-centered differencing discretization of Eqs. (14) and (13): the discretization is stable, but in order to obtain the expected accuracy, the time step $\Delta t$ needs to satisfy the condition $c \Delta t / \Delta x<1$. The acoustic pressure $\delta$ contains long-wavelength waves, with the largest wavelength on the order of $1 / M a$. For low Mach number flow, there is no hope of resolving such long wavelengths in our box of length 1 , and we expect the convergence rate for $\delta$ to be poor, as seen in Table VI. The long-wavelength acoustic waves have very little feedback to the incompressible flow at small Mach number. As $P$ decreases, the corresponding Mach number increases, and it becomes more and more important that the acoustic waves be resolved. For $P=100$ and 10, however, the condition $c \Delta t / \Delta x<1$ for the accuracy of the backward Euler-centered differencing scheme is not met, and the feedback of the acoustic waves to the flow is not well-resolved; thus the accuracy of the overall scheme deteriorates. For $P=1$, the largest wavelength is on the order of the size of the box, which means that

\section{TABLE II}

\section{$L^{1}$ Convergence Results-Density $\rho$}

\begin{tabular}{rccccc}
\hline$P_{0}$ & $32-64$ & Rate & $64-128$ & Rate & $128-256$ \\
\hline 1 & $7.374 \mathrm{e}-3$ & 1.08 & $3.491 \mathrm{e}-3$ & 1.05 & $1.684 \mathrm{e}-3$ \\
10 & $3.551 \mathrm{e}-3$ & 0.69 & $2.192 \mathrm{e}-3$ & 0.77 & $1.280 \mathrm{e}-3$ \\
100 & $3.745 \mathrm{e}-4$ & 0.18 & $3.275 \mathrm{e}-4$ & n/a & $3.898 \mathrm{e}-4$ \\
1000 & $3.675 \mathrm{e}-4$ & 2.07 & $8.726 \mathrm{e}-5$ & 2.00 & $2.781 \mathrm{e}-5$ \\
\hline
\end{tabular}




\section{TABLE III}

$L^{1}$ Convergence Results-Incompressible Velocity $u_{d}$

\begin{tabular}{rccccc}
\hline$P_{0}$ & $32-64$ & Rate & $64-128$ & Rate & $128-256$ \\
\hline 1 & $3.383 \mathrm{e}-3$ & 1.54 & $1.169 \mathrm{e}-3$ & 1.23 & $5.003 \mathrm{e}-4$ \\
10 & $3.666 \mathrm{e}-3$ & 1.88 & $9.508 \mathrm{e}-4$ & 1.70 & $2.928 \mathrm{e}-4$ \\
100 & $3.808 \mathrm{e}-3$ & 2.07 & $9.047 \mathrm{e}-4$ & 2.03 & $2.239 \mathrm{e}-4$ \\
1000 & $3.836 \mathrm{e}-3$ & 2.03 & $9.422 \mathrm{e}-4$ & 2.00 & $2.353 \mathrm{e}-4$ \\
\hline
\end{tabular}

TABLE IV

$L^{1}$ Convergence Results-Incompressible Pressure $\pi$

\begin{tabular}{rcrccc}
\hline \multicolumn{1}{r}{$P_{0}$} & $32-64$ & Rate & $64-128$ & Rate & $128-256$ \\
\hline 1 & $3.767 \mathrm{e}-3$ & .73 & $2.263 \mathrm{e}-3$ & 0.88 & $1.232 \mathrm{e}-3$ \\
10 & $1.787 \mathrm{e}-3$ & .33 & $1.415 \mathrm{e}-3$ & 0.58 & $9.522 \mathrm{e}-3$ \\
100 & $2.595 \mathrm{e}-3$ & 1.28 & $1.074 \mathrm{e}-3$ & 0.17 & $9.563 \mathrm{e}-4$ \\
1000 & $2.342 \mathrm{e}-3$ & 1.95 & $6.071 \mathrm{e}-4$ & 1.79 & $1.761 \mathrm{e}-4$ \\
\hline
\end{tabular}

TABLE V

$L^{1}$ Convergence Results-Total Velocity $u$

\begin{tabular}{rccccc}
\hline \multicolumn{1}{r}{$P_{0}$} & $32-64$ & Rate & $64-128$ & Rate & $128-256$ \\
\hline 1 & $5.517 \mathrm{e}-3$ & 1.29 & $2.262 \mathrm{e}-3$ & 1.11 & $1.047 \mathrm{e}-2$ \\
10 & $6.404 \mathrm{e}-3$ & 0.86 & $3.529 \mathrm{e}-3$ & 0.78 & $2.050 \mathrm{e}-2$ \\
100 & $3.898 \mathrm{e}-4$ & 1.70 & $1.195 \mathrm{e}-3$ & 0.14 & $1.087 \mathrm{e}-3$ \\
1000 & $3.836 \mathrm{e}-3$ & 2.02 & $9.426 \mathrm{e}-4$ & 1.87 & $2.575 \mathrm{e}-4$ \\
\hline
\end{tabular}

TABLE VI

$L^{1}$ Convergence Results-Acoustic Pressure $\delta$

\begin{tabular}{rccccc}
\hline \multicolumn{1}{r}{$P_{0}$} & $32-64$ & Rate & $64-128$ & Rate & $128-256$ \\
\hline 1 & $6.599 \mathrm{e}-3$ & 1.10 & $3.080 \mathrm{e}-3$ & 1.09 & $1.446 \mathrm{e}-3$ \\
10 & $4.062 \mathrm{e}-2$ & 0.53 & $2.812 \mathrm{e}-2$ & 0.70 & $1.720 \mathrm{e}-2$ \\
100 & $1.588 \mathrm{e}-2$ & $\mathrm{n} / \mathrm{a}$ & $3.986 \mathrm{e}-2$ & $\mathrm{n} / \mathrm{a}$ & $4.952 \mathrm{e}-2$ \\
1000 & $2.809 \mathrm{e}-4$ & n/a & $4.158 \mathrm{e}-4$ & n/a & $2.655 \mathrm{e}-3$ \\
\hline
\end{tabular}

\section{TABLE VII}

$L^{1}$ Convergence Results for the "Incompressible Variables"

\begin{tabular}{llllll}
\multicolumn{7}{c}{ at $\boldsymbol{t}=\mathbf{0 . 5}$ for $\boldsymbol{P}=\mathbf{1 0 0 0}$} \\
\hline & $32-64$ & Rate & $64-128$ & Rate & $128-256$ \\
\hline$\rho$ & $1.930 \mathrm{e}-3$ & 2.03 & $4.712 \mathrm{e}-4$ & 2.03 & $1.163 \mathrm{e}-4$ \\
$u_{d}$ & $4.659 \mathrm{e}-3$ & 2.09 & $1.093 \mathrm{e}-3$ & 2.04 & $2.653 \mathrm{e}-4$ \\
$\pi$ & $2.940 \mathrm{e}-3$ & 2.20 & $6.377 \mathrm{e}-4$ & 1.90 & $1.711 \mathrm{e}-4$ \\
\hline
\end{tabular}


most of the long-wavelength components of the solution will be resolved, and we expect to get first-order accuracy.

The $P=10$ case represents a transition of the numerical method from a second-order one to a first-order one. Although the large-wavelength acoustic waves in $\delta$ are still not quite resolved, we can see that the "compressible flow" quantities $\rho, u$, and $\delta$ are beginning to show convergent behavior. For $P=1$, our scheme is clearly first-order accurate, with the backward Euler-centered differencing fully resolving all features of the acoustic waves.

For $P=1000$, we surmised that the backward Euler-centered differencing Helmholtz solver would eventually damp out the acoustic waves so that the feedback would be negligible. We let the $P=1000$ run go up to $t=0.5$. The convergence rates for $\rho, u_{d}$, and $\pi$ in this case are presented in Table VII. These rates are in agreement with the results of Bell and Marcus [20]. These rates are an improvement over those at $t=0.125$, indicating that the long-wavelength components have indeed sufficiently decayed that they have no effect on the convergence rates.

Our next convergence test is to study the behavior of the numerical method in a potential flow. We would expect only first-order convergence, because backward Euler-centered difference is only first-order accurate. Initially, we have an adiabatic, quiescent flow

$$
\begin{aligned}
& u(x, y, 0)=v(x, y, 0)=0.0 \\
& \rho(x, y, 0)=1-\frac{1}{4} \tanh \left(x-\frac{1}{2}\right) \tanh \left(y-\frac{1}{2}\right) \\
& p(x, y, 0)=\rho(x, y, 0)^{\gamma}
\end{aligned}
$$

on the unit square $0 \leq x \leq 1,0 \leq y \leq 1$. Again, the spatial and temporal discretizations are $\Delta x=\Delta y=1 / 25,1 / 50,1 / 100,1 / 200 ; \Delta t=0.5 \Delta x /|c|_{\infty}$. Here $|c|_{\infty}$ is set to 2.0. The convergence rates for the density $\rho$, the pressure $\delta$, the velocity $\mathbf{u}_{p}$, and the quantity $p / \rho^{\gamma}$ at time $t=1$ are presented in Table VIII.

In this test problem, only low-frequency waves are present. Time step size is also sufficiently small. In these circumstances, the Helmholtz solver is first-order accurate, and our numerical results are first-order convergent for the "potential" variables.

Next we present computational results of flow in a converging nozzle. In this flow, we prescribe an inflow velocity of $u_{\text {in }}$ at $x=-1$, and the total pressure is constant $P_{\text {out }}$ at the outflow at $x=3.0$. The initial density $\rho$ is $\rho_{0}$, and the density at inflow is held constant at $\rho_{0}$. The nozzle walls are $y=0$ and $y=1 / 4(3-\tanh (4(x-4)))$. The grid is $100 \times 25$ cells, as shown in Fig. 1 . In our experiments, we fix $P_{\text {out }}=100$ and $\rho_{0}=1.0$, and let $u_{\text {in }}=1.0,2.0,3.0,4.0,5.0$. All are executed up to $t=3.0$. Table IX shows the approximate maximum Mach numbers in the duration of each computation.

Results for $u_{\text {in }}=4.0$ at time $t=3.0$ are shown in Figs. 2-8. As the fluid flows past the bend on the top wall, it is compressed. This compressibility effect may be seen from

\section{TABLE VIII}

\section{$L^{1}$ Convergence Results for the Potential Flow}

\begin{tabular}{lccccc}
\hline & $32-64$ & Rate & $64-128$ & Rate & $128-256$ \\
\hline$\rho$ & $6.179 \mathrm{e}-4$ & 0.92 & $3.266 \mathrm{e}-4$ & 0.86 & $1.801 \mathrm{e}-4$ \\
$\delta$ & $6.221 \mathrm{e}-4$ & 0.81 & $3.554 \mathrm{e}-4$ & 0.81 & $2.032 \mathrm{e}-4$ \\
$\left|u_{p}\right|$ & $6.092 \mathrm{e}-4$ & 0.99 & $3.065 \mathrm{e}-4$ & 0.96 & $1.575 \mathrm{e}-4$ \\
$p / \rho^{\gamma}$ & $3.421 \mathrm{e}-5$ & 1.04 & $1.661 \mathrm{e}-5$ & 0.99 & $8.393 \mathrm{e}-6$ \\
\hline
\end{tabular}


TABLE IX

Maximum Mach Numbers $M a$ as a Function of Inlet Velocity $u_{\text {in }}$

\begin{tabular}{cc}
\hline$u_{\text {in }}$ & Approx. $M a$ \\
\hline 1 & 0.12 \\
2 & 0.26 \\
3 & 0.42 \\
4 & 0.60 \\
5 & 0.80 \\
\hline
\end{tabular}

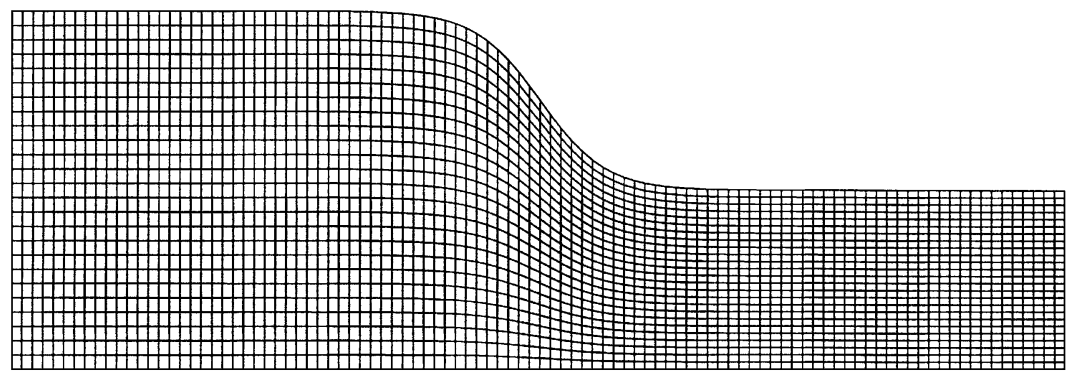

$\mathrm{x}$

FIG. 1. The grid used in the converging channel computation.

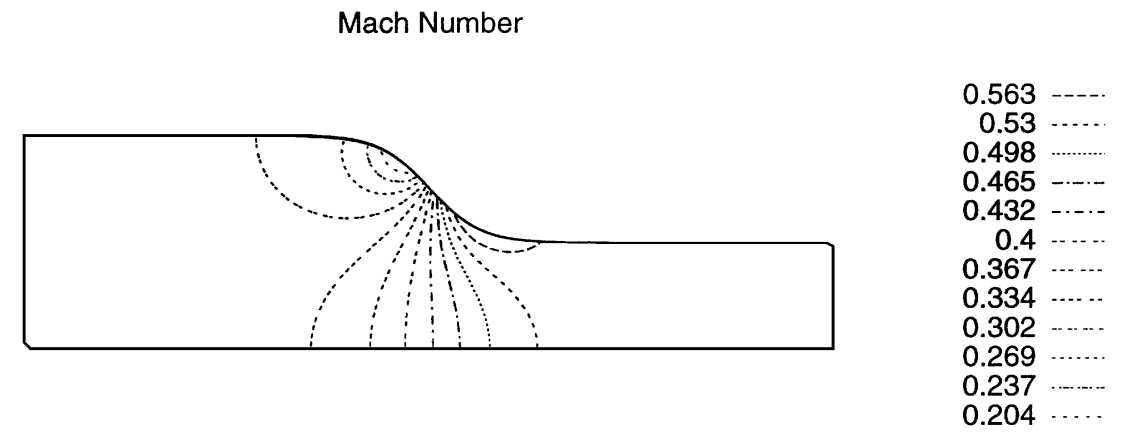

FIG. 2. The Mach number $M a$ at $t=3.0 . u_{\text {in }}=4.0$.

Acoustic Pressure

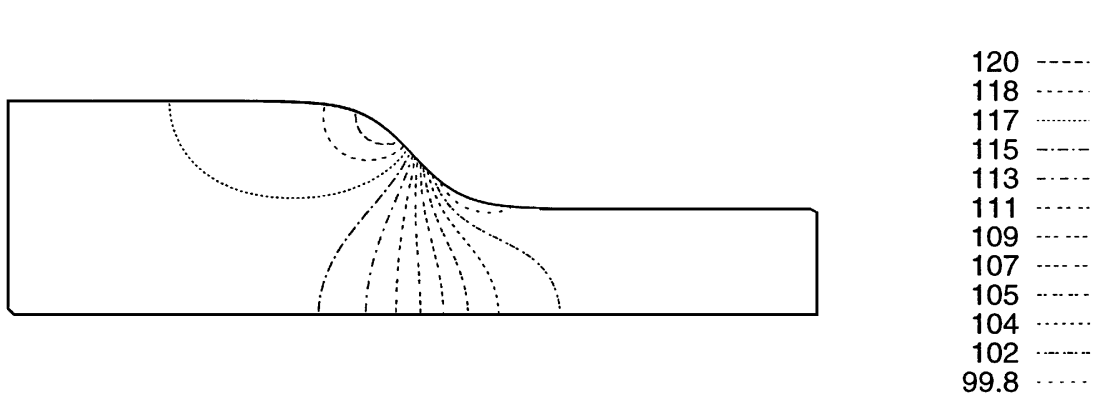

FIG. 3. The acoustic pressure $\delta$ at $t=3.0 . u_{\text {in }}=4.0$. 
Total Velocity u

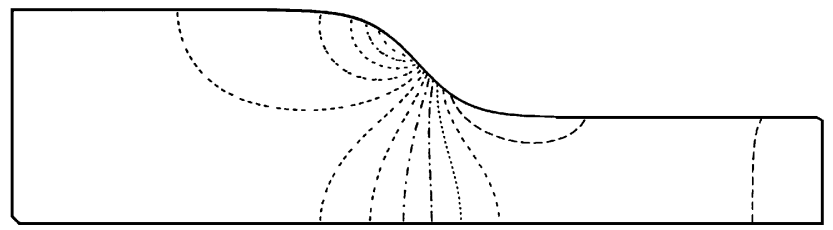

$5.16 \cdots$

4.75

FIG. 4. The total $x$-velocity $u$ at $t=3.0 . u_{\text {in }}=4.0$.

\section{Total Velocity v}

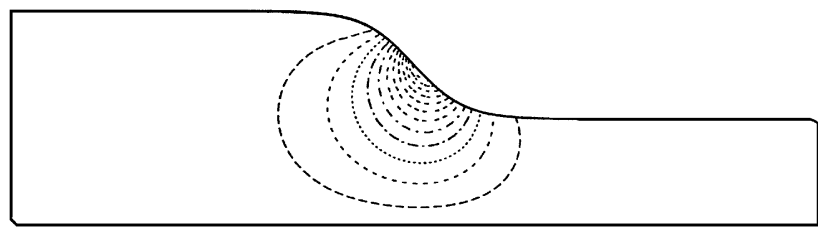

FIG. 5. The total $y$-velocity $v$ at $t=3.0 . u_{\text {in }}=4.0$.

Incompressible Pressure

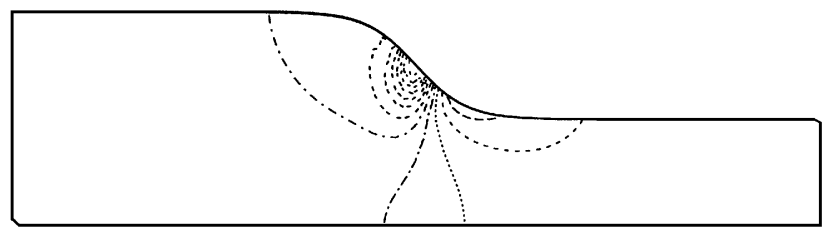

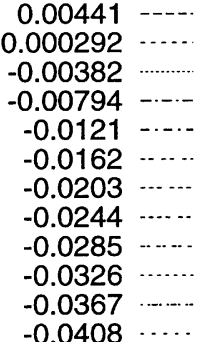

FIG. 6. The incompressible pressure $\pi$ at $t=3.0 . u_{\text {in }}=4.0$.

Incompressible Velocity ud

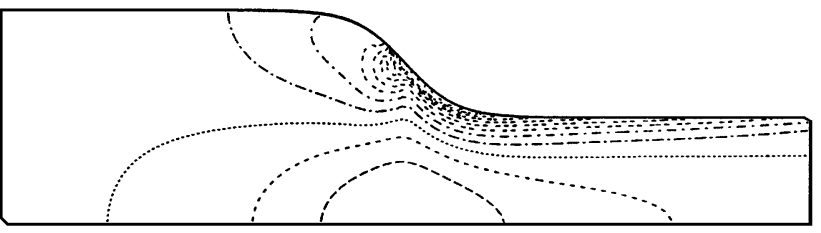

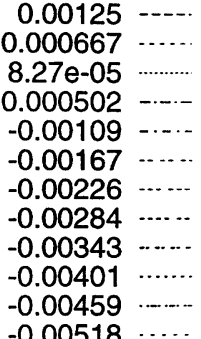

FIG. 7. The incompressible $x$-velocity $u_{d}$ at $t=3.0 . u_{\text {in }}=4.0$. 
Incompressible Velocity vd

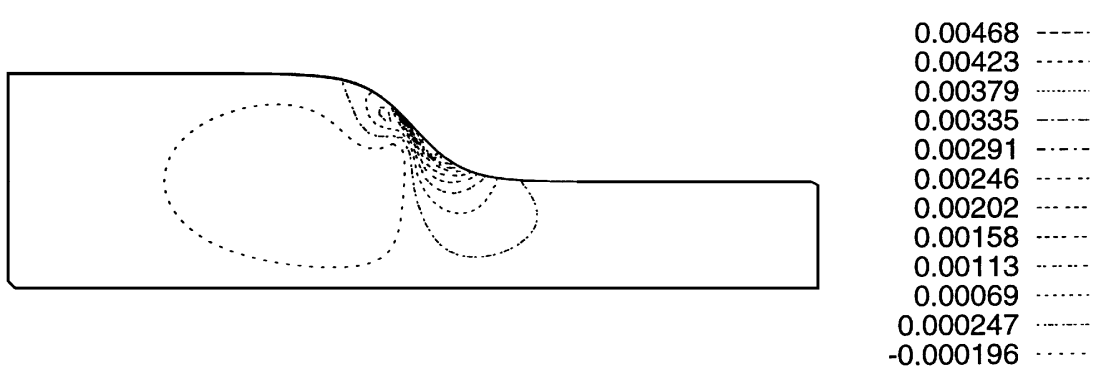

FIG. 8. The incompressible $y$-velocity $v_{d}$ at $t=3.0 . u_{\text {in }}=4.0$.

$u_{\text {in }}=1.0$ on and up. The total pressure is mostly acoustic pressure $\delta$. The pressure increases in time gradually, eventually becoming almost constant from the inlet to the beginning of the bend, decreasing rapidly as the fluid traverses the bend, and becoming constant again as the fluid flows out of the channel. A high-pressure spot is found near the top of the bend, while a low-pressure spot can be found near the bottom of the bend. The pressure profiles are basically the same for all Mach numbers (except when the Mach number becomes close to 1; see below); when the Mach number is greater, the pressure drop across the bend is greater, as expected. The total velocity $\mathbf{u}$ is mostly potential by design. The $x$-component of the total velocity, $u$, settles into a pattern of low, almost constant in space upstream, rapid acceleration through the high pressure gradient region, and high, almost constant in space downstream. The velocity gradient is greater for greater values of inflow velocity. The $y$-component of the total velocity, $v$, remains "localized" around the bend for all Mach numbers. The incompressible quantities $\pi$ and $\mathbf{u}_{d}$ are generally small after the initialization effects are allowed to decay sufficiently; as the Mach number increases, their magnitudes also increase.

As we increase the inflow velocity, we shall eventually reach a sonic point near outflow. Our algorithm and boundary conditions are not expected to handle sonic or supersonic flows. Generally, when the Mach number becomes too large (when $u_{\text {in }} \geq 5$ in these experiments), the computation gives poor results, especially near the bend, where a "separation" is observed. If sonic is reached in the course of computation, the computation will eventually break down. These will be topics for future investigations.

\section{CONCLUSIONS}

We derived the evolution equations for the divergence-free and the curl-free parts of the velocity field in an inviscid, compressible flow. A numerical method for simulating flows whose Mach numbers are less than one based on these equations has been devised. Convergence results show that our numerical method is second-order accurate for lowMach-number flows and first-order accurate for higher Mach numbers. Results of other numerical experiments were presented.

One area of improvement is the resolution of acoustic waves. As we have seen from our convergence tests, there can be important feedback from the acoustics into the flow even at low Mach numbers. While it may not be important that the sound waves be resolved, we do wish to minimize the polluting effects of the poor resolution of acoustics. A possible 
approach would be to use a higher-order time discretization in the Helmholtz solve. A desirable higher-order time discretization should contain enough damping to eliminate acoustic waves effectively at very small Mach numbers, and not as much damping for the sound waves at higher frequencies.

Another area of improvement lies in the numerical implementation of boundary conditions. We do not yet fully understand how to implement boundary conditions numerically in general; each case has to be "engineered" to obtain physically reasonable results. We would like to develop a consistent framework for the application and the implementation of boundary conditions for the decomposed velocity fields, as well as the corresponding boundary conditions for the projection operators.

Finally, we would like to extend our formulation to include viscosity and chemistry terms to study combustion applications.

\section{REFERENCES}

1. E. S. Oran, Numerical simulation of unsteady combustion, in Advances in Combustion Science: In Honor of Ya. B. Zeldovich, Prog. Astronau. Aeronaut. 173, 179 (1997).

2. M. J. Lighthill, On sound generated aerodynamically. I. General theory, Proc. R. Soc. London Ser. A 211, 564 (1952).

3. F. Harlow and A. Amsden, Numerical calculation of almost incompressible flow, J. Comput. Phys. 3, 80 (1968).

4. V. Casulli and D. Greenspan, Pressure method for the numerical solution of transient, compressible fluid flows, Int. J. Numer. Methods Fluids 4, 1001 (1984).

5. G. Patnaik, R. H. Guirguis, J. P. Boris, and E. S. Oran, A barely implicit correction for flux-corrected transport, J. Comput. Phys. 71, 1 (1987).

6. S. Abarbanel, P. Duth, and D. Gottlieb, Splitting methods for low Mach number Euler and Navier-Stokes equations, Comput. \& Fluids 17, 1 (1989).

7. B. Gustafsson and H. Stoor, Navier-Stokes equations for almost incompressible flow, SIAM J. Numer. Anal. 28, 1523 (1991).

8. J. Sesterhenn, B. Mueller, and H. Thomann, Flux-vector splitting for compressible low Mach number flow, Comput. \& Fluids 22, 441 (1993).

9. R. Klein, Semi-implicit extension of a Godunov-type scheme based on low Mach number asymptotics. I. One-dimensional flow, J. Comput. Phys. 121, 213 (1995).

10. C.-D. Munz, S. Roller, R. Klein, and K. J. Geratz, The extension of incompressible flow solvers to the weakly compressible regime, preprint (1997).

11. K. Pao, and C. Anderson, Computation of Flow Noise in Two Dimensions, Los Alamos National Laboratory Technical Report LA-UR-94-1354 (1994). [unpublished]

12. S. Klainermann and A. Majda, Compressible and incompressible fluids, Comm. Pure Appl. Math. 35, 629 (1982).

13. H. O. Kreiss, J. Lorenz, and M. Naughton, Convergence of the solutions of the compressible to the solutions of the incompressible Navier-Stokes equations, Adv. Appl. Math. 12, 187 (1991).

14. A. Chorin and J. E. Marsden, A Mathematical Introduction to Fluid Mechanics (Springer-Verlag, New York, 1979).

15. C. Anderson, Derivation and Solution of the Discrete Pressure Equations for the Incompressible Navier Stokes Equations, University of California, Los Angeles, Computational and Applied Mathematics Report 88-36 (1998). [unpublished]

16. F. Harlow and J. E. Welch, Numerical calculations of time dependent viscous incompressible flow with free surface, Phys. Fluids 8, 2182 (1965).

17. P. Colella, A multidimensional second order Godunov scheme for conservation laws, J. Comput. Phys. 87, 171 (1990). 
18. B. van Leer, Toward the ultimate conservative difference scheme: A second-order sequel to Godunov's method, J. Comput. Phys. 32, 101 (1979).

19. J. Bell, P. Colella, and H. Glaz, A second order projection method for the incompressible Navier-Stokes equations, J. Comput. Phys. 85, 257 (1989).

20. J. Bell and D. Marcus, A second order projection method for variable-density flows, J. Comput. Phys. 101, 334 (1992).

21. M. Lai, A Projection Method for Reacting Flow in the Zero Mach Number Limit, Ph.D. thesis, University of California, Berkeley, 1993. [unpublished] 